\title{
Estrategias para la evaluación en educación a distancia: un análisis de las opciones empleadas en el programa de educación general básica de la UNED
}

Jenny Bogantes Pessoa

Cátedra Didáctica del Lenguaje, Universidad Estatal a Distancia, (UNED). Costa Rica; jbogantes@uned.ac.cr

Recibido: 15 de octubre del 2014

Corregido: 03 de marzo del 2015

Aceptado: 31 de julio del 2015

\section{Resumen}

El artículo expone algunos los hallazgos de la investigación que se llevó a cabo gracias al financiamiento de la Coordinación Educativa y Cultural Centroamericana (CECC) SICA); se analizaron las opciones empleadas en la evaluación de los aprendizajes de nueve asignaturas pertenecientes al Programa de Educación General Básica I y II Ciclos, de la Escuela Ciencias de la Educación en la Universidad Estatal a Distancia de Costa Rica (UNED). Se empleó una metodología de investigación mixta; en esta entrega se brindan los resultados correspondientes al primer objetivo de la investigación que responde a la etapa cuantitativa: "Identificar la índole de opciones evaluativas que se emplean en las asignaturas para recopilar las evidencias de aprendizaje del estudiantado"; se examinaron documentos pertenecientes a dos periodos académicos y se logró determinar que en la mayoría de los casos se evalúa por medio de pruebas escritas, tareas o proyectos que incluyen al menos un instrumento de evaluación como, por ejemplo, ensayo, entrevista, cuadro comparativo o reporte de observación.

Palabras clave: Calidad de la educación, educación a distancia, formación de docentes de primaria, educación superior, evaluación del currículo.

\begin{abstract}
Assessment strategies in distance education: a review of the options used in the general basic education program at the UNED

This paper reports the findings of a research conducted thanks to Educational and Cultural Coordination (CECC) SICA) funding; the evaluation options used by nine subjects belonging to the General Basic Education Program, first and second cycles, of the Education Sciences School in the State Distance University of Costa Rica (UNED) were analyzed. A mixed research methodology was followed; the results for the first research's objective, that responds to the quantitative stage are presented: "Identifying the nature of evaluative options used in the subjects to gather evidence of student learning"; documents belonging to two academic periods were examined, in which it was determined that in most cases is assessed through written tests and homework or projects that include at least an assessment tool such as essay, interview, comparative table or observation report.
\end{abstract}

Keywords: Education quality, distance education, elementary teacher education, higher education, curriculum evaluation. 


\section{INTRODUCCIÓN}

La sociedad del siglo XXI se caracteriza por ser un periodo de cambio constante. Las personas se desenvuelven en un mundo globalizado y están habituadas al confort que brinda la modernidad. La educación no es la excepción; por el contrario, debe ir adaptándose a las innovaciones para así responder a las exigencias actuales. Ahora bien, como parte esencial del campo educativo, la evaluación de los aprendizajes debe avanzar y mejorar de manera tal que logre evidenciar el aprendizaje obtenido por las personas durante el proceso educativo.

Desafortunadamente, la evaluación se ha convertido en el talón de Aquiles de la educación, debido a que aún en el nivel universitario se pueden encontrar grandes deficiencias en el acto evaluativo. En la mayoría de las ocasiones se habla acerca de la enseñanza por medio del constructivismo y de cómo el estudiante debe ser el centro del aprendizaje; a pesar de ello, se continúa evaluando de manera tradicional mediante pruebas escritas principalmente; las cuales no siempre cuentan con la validez y confiabilidad que se requiere $y$, por lo general, no logran comprobar el aprendizaje, sino que se convierten en un acto memorístico de "recitación" de contenidos.

El tema evaluativo de la formación docente en un sistema de enseñanza superior a distancia se constituye como elemento de mayor interés y en un gran reto debido a la dificultad que implica; por ende, el modelo de evaluación que se emplee debe ser lo suficientemente eficaz como para valorar el desempeño académico en las condiciones mencionadas.

La UNED ha procurado ofrecer un marco teórico denominado Modelo Pedagógico, con el propósito de guiar el aprendizaje y los procesos evaluativos que se realicen en las diferentes carreras que se imparten dentro de la institución.

Los sistemas a distancia plantean el reto de cómo lograr un modelo pedagógico que permita a la evaluación convertirse en un medio que realimente al estudiantado y al docente en su quehacer diario. Una respuesta a este dilema ha sido recurrir a la enseñanza y la evaluación por medio de plataformas en línea, propuesta que forma parte de los nuevos retos que se han planteado en la universidad; sin embargo, en un primer acercamiento al quehacer universitario se puede notar que la evaluación presencial muestra un gran peso en la calificación final.

En la Escuela Ciencias de la Educación (ECE) de la UNED, la evaluación es un tema prioritario ya que no solo certificará el aprendizaje sino que servirá de ejemplo para que el estudiantado logre mejorar y aplicar estos conocimientos en su futuro desarrollo como profesionales. Con base en lo anterior, surge la necesidad de investigar la evaluación aplicada en el Programa de Diplomado, Bachillerato y Licenciatura en I y II Ciclos de la Enseñanza General Básica de la Universidad Estatal a Distancia para identificar la índole de opciones evaluativas que se utilizan en las asignaturas programa. La índole de opciones evaluativas se entiende como la variedad de instrumentos empleados en una asignatura para evaluar el aprendizaje durante un periodo académico determinado.

Por el motivo anterior, en el año 2011 se decide participar en el cartel de invitación que presenta la Coordinación Educativa y Cultural Centroamericana (CECC/SICA) y se obtienen los fondos de la cooperación holandesa para realizar una investigación dentro del proyecto Mejoramiento de las condiciones académicas y técnicas que se llevan a cabo en los procesos de formación inicial de docentes para atender la educación inicial y la educación primaria o básica en la región de Centroamérica y la República Dominicana (FID-Holanda 2010-2012).

Mediante la investigación se obtendría información acerca de las propuestas de evaluación de los aprendizajes presentes en las asignaturas del programa en mención y su concordancia con los objetivos planteados en el documento Modelo Pedagógico. Con este fin se planteó la aplicación de una metodología de investigación mixta, mediante la que se analizaron las pruebas escritas, las orientaciones académicas (en las que se incluyen los instrumentos de evaluación como tareas, proyectos, observaciones, entre otros) y las descripciones curriculares de las asignaturas que se seleccionaron como muestra.

Además, en la etapa cualitativa se aplicaron cuestionarios a las personas tutoras y se entrevistó individualmente a las personas encargadas de las cátedras con el propósito de conocer su percepción sobre la evaluación de los aprendizajes. 


\section{REFERENTE TEÓRICO}

\section{- Concepto de evaluación de los aprendizajes}

La evaluación constituye uno de los temas con mayor protagonismo en el ámbito educativo, no porque se trate de un tema nuevo en absoluto, sino debido a que toda la sociedad en su conjunto es más reflexiva acerca de la importancia y las repercusiones de evaluar o de ser evaluado, en tanto la asimilación de contenidos y destrezas resulta vital en el mundo de la información. Existe, quizás, una mayor conciencia de la necesidad de alcanzar determinadas cotas de calidad educativa, de aprovechar adecuadamente los recursos, el tiempo y los esfuerzos; por otra parte, el nivel de competencia entre las personas y las instituciones también es mayor. En ese sentido, Álvarez, González, González, Muñiz, Núñez, Rodríguez y Soler (2006, p. 47)

En el sistema educativo, la evaluación ha de servir también para recabar y analizar datos respecto de sus elementos relevantes: objetivos, medios que se utilizan, tareas que se llevan a cabo en el aula, relaciones de comunicación que se establecen, organización e incluso la propia evaluación que se realiza.

Uno de los factores más importantes, en tanto explican por qué ocupa actualmente un lugar tan destacado, es la comprensión por parte de los profesionales de la educación de que es la evaluación quien en realidad prescribe y decide de facto el qué, cómo, por qué y cuándo enseñar. Es decir, las decisiones que se hayan tomado durante los procesos de enseñanza aprendizaje.

La evaluación debe realizarse durante todo el acto educativo, y considerar siempre sus funciones diagnóstica, formativa y sumativa; a pesar de eso, existe una visión muy arraigada en el personal docente que insiste en concebirla como un elemento final en el proceso de aprendizaje con la aplicación de pruebas escritas. De hecho, Moreno (2009, p. 575) menciona:

Aun cuando los exámenes teóricos más recientes exaltan una evaluación integral, que incluye variadas técnicas para la recolección de información sobre el desempeño del alumno y que se valoren diversos aspectos de su actuación, el discurso de los alumnos revela justo lo contrario. El examen escrito convencional sigue siendo la técnica privilegiada y la de mayor peso en la evaluación. A la hora de la verdad, lo que realmente cuenta es el resultado obtenido en el examen y no el proceso.

En general, uno de los objetivos prioritarios del estudiantado es satisfacer las exigencias de los "exámenes". Así, Álvarez y otros (2006, p.50), en el marco de la tendencia cuantitativa de la evaluación, concluyen:

Los exámenes, y en general los instrumentos de la evaluación, ayudan a calificar, y la calificación pretende ser un resumen de los resultados de un proceso de aprendizaje, es decir, la valoración del estado interno del alumno, la medida de lo que es, sabe y puede hacer, indispensable para una toma de decisiones adecuada.

Por lo tanto, la evaluación, al prescribir realmente los objetivos de la educación, determina en gran medida lo que el estudiantado aprende y cómo lo aprende, lo que el profesorado enseña y cómo lo enseña, los contenidos, los métodos y los recursos pedagógicos; en otras palabras, el producto y el proceso de la educación. Se quiera o no, de forma consciente o inconsciente, la actividad educativa de estudiantes y de docentes se encuentra en algún modo canalizada por la evaluación.

La concepción de evaluación de los aprendizajes ha ido evolucionando hacia un concepto más integral, evaluar para aprender; de esa manera, la docencia se orienta hacia el aprendizaje y la evaluación deja de reducirse a una mera calificación para convertirse en un proceso que contribuye a optimizar los aprendizajes. En palabras de Bordas y Cabrera, citados por Padilla y Gil (2008): "La evaluación deber ser un proceso reflexivo donde el que aprende toma conciencia de sí mismo y de sus metas y el que enseña se convierte en guía que orienta hacia el logro de unos objetivos culturales $y$ formativos" (p. 468).

El cambio en la concepción de la evaluación ha transformado también la manera de entenderla y de practicarla, de forma tal que promueve el empleo de diversas estrategias de evaluación, diferentes al tradicional examen escrito. La evaluación se transforma en un mecanismo de orientación del aprendizaje centrado en un proceso que ayuda al alumnado a aprender. Razón por la cual, autores como Morales (2006), citado 
por Padilla y Gil (2008, p.469) han sugerido la necesidad de replantear el lugar asignado a la evaluación: "Pensar en ella desde el principio, cómo la planificamos, cómo preguntamos, qué tareas proponemos... de manera que sea coherente con los objetivos de aprendizaje y se convierta en la pieza clave para mejorar la enseñanza (diseñar ejercicios, etcétera.)".

La evaluación es inherente al aprendizaje, debe darse de manera integral y continua durante el proceso de aprendizaje; además permite analizar y tomar decisiones oportunas en el proceso educativo.

\section{Evaluación de los aprendizajes en los modelos de educación a distancia}

La educación a distancia es un paradigma que en los últimos años ha surgido con bastante fuerza, sobre todo porque brinda la posibilidad de llevar educación superior a poblaciones que no tienen acceso al sistema presencial, ya sea por lejanía o imposibilidad, por factores laborales o de transporte. Esta nueva realidad educativa ha originado que las teorías tradicionales de la educación, en lo referente a estrategias didácticas y técnicas de evaluación, sean replanteadas en términos de las características de la población estudiantil y de los modelos pedagógicos emergentes.

La aparición de modelos de enseñanza aprendizaje a distancia plantean diversas interrogantes: ¿Cuáles características deben mostrar los instrumentos de evaluación? ¿Tiene sentido la evaluación presencial? ¿Cómo incorporar la evaluación en línea (sistemas virtuales)? ¿Se puede lograr una evaluación formativa utilizando modelos evaluativos no presenciales? ¿Pueden los modelos tradicionales de evaluación ser transferidos a sistemas educativos a distancia?

En el caso de la Universidad Estatal a Distancia, la evaluación de los aprendizajes está caracterizada en el modelo pedagógico, de modo que su planteamiento constituye el principal referente para las propuestas evaluativas de las asignaturas en las diferentes carreras que se imparten en la UNED y desde luego es parte de lo que las personas responsables de los distintos programas y de las cátedras deben analizar y discutir con sus personas tutoras. Este modelo fue aprobado por el Consejo Universitario en sesión 1714-2004 de julio de
2004 y los aspectos más relacionados con esta temática son los siguientes:

- La evaluación de los aprendizajes debe estar centrada en el estudiante, por tanto, no basta con recolectar información, conceptos o procedimientos en un momento dado, es decir, lo que el estudiante logró memorizar.

- Los instrumentos de evaluación de los aprendizajes y las formas cómo se apliquen, deben ser válidos y transparentes, es decir, en cuanto a la primera responder a criterios técnicos específicos y en cuanto a la segunda ser elaborados a través de procedimientos claros, honestos y ágiles.

- En las propuestas evaluativas se debe fomentar y valorar la capacidad del estudiante para generar ideas propias y no apoyar exclusivamente a un autor o a las expuestas en el material didáctico de cada asignatura.

El modelo pedagógico centrado en el estudiantado visualiza la importancia de desarrollar una concepción metodológica más abierta, flexible, que le ofrezca herramientas para construir su propio proceso de aprendizaje y lo haga protagonista en la apropiación del conocimiento, por medio de una concepción de evaluación como regulación y autorregulación de los aprendizajes. Asimismo indica:

La evaluación así entendida se integra al proceso de enseñar y de aprender a distancia y deja de ser un momento final, separado e independiente. Se convierte en el elemento que da dirección y sentido al diseño de materiales, a la programación de cursos, a la acción de facilitación y apoyo al aprendizaje y, en última instancia, a la formación de un estudiante autónomo y capaz de seguir aprendiendo solo. (UNED, 2004, p. 40)

Se puede agregar que dentro de las acciones que se llevan a cabo en la UNED para asesorar y capacitar en materia de evaluación de los aprendizajes a los distintos programas de la universidad, se puede mencionar la creación de la Comisión Institucional de Evaluación de los Aprendizajes en el año 2007, integrada por personas representantes de las cuatro escuelas, de extensión, posgrado y del Programa Apoyo Curricular y Evaluación de los Aprendizajes (PACE). Esta comisión se dio a la tarea de elaborar, entre otros, los documentos titulados 
Técnicas y herramientas para la evaluación alternativa y Lineamientos para la evaluación de los aprendizajes en la Universidad Estatal a Distancia.

En el primer documento se ofrece a las personas evaluadoras una serie de características y consideraciones para la utilización de técnicas y herramientas de evaluación alternativa; además, se menciona que las técnicas convencionales de evaluación han sido insuficientes para trascender la función sumativa, haciéndose necesario recurrir al empleo de metodología no tradicional acorde con el paradigma constructivista para que el estudiantado desarrolle su proceso de aprendizaje. Mediante el documento de lineamientos, se les brinda una serie de recomendaciones acerca de técnicas alternativas de evaluación, estructura básica para la elaboración del solucionario, directrices para la confección de pruebas escritas y para las orientaciones académicas.

Finalmente, la temática se ve reflejada en el Plan Académico Institucional 2008-2011, específicamente en el objetivo 7: "Ejecutar procesos de evaluación y de autoevaluación que respalden el aprendizaje autónomo y la excelencia académica", para el cual se apuntan acciones como:

7.3 Elaboración de un plan de mejoramiento de los procesos de evaluación de los aprendizajes en los diferentes niveles de formación.

7.4 Aplicación, seguimiento y valoración de diversas modalidades de evaluación de los aprendizajes que favorezcan la autoevaluación y coevaluación entre los estudiantes, como una construcción personal y participativa de aprender a aprender.

7.5 Evaluación de la calidad de los instrumentos de evaluación de los diferentes cursos por año. (p. 51)

Como puede notarse, la evaluación constituye el eje del aprendizaje en todo el modelo pedagógico, de manera que para el caso de la ECE, la evaluación debe convertirse en la vía para seguir, reorientar, corregir y estimular el aprendizaje.

\section{METODOLOGÍA Y MATERIALES}

La investigación se llevó a cabo mediante el enfoque mixto; de acuerdo con Hernández, S., Fernández,
C. y Baptista, P. (2006) el propósito de este enfoque es recolectar, analizar y vincular datos cuantitativos y cualitativos en un mismo estudio para responder al planteamiento del problema.

\section{- Los instrumentos}

Se realizaron dos etapas, sin embargo solamente la primera etapa se relaciona con el presente artículo:

Etapa cuantitativa: Se emplearon instrumentos para identificar la índole de propuestas evaluativas aplicadas en las asignaturas y para analizar técnicamente las pruebas escritas aplicadas en el 2011 y 2012, de la siguiente manera:

Técnica: Revisión documental.

Instrumentos: Guías de revisión documental para

Orientaciones Académicas y para pruebas escritas.

Etapa cualitativa: Se buscaba caracterizar los instrumentos y técnicas de evaluación que se aplicaron en el 2011 y 2012, indagar la concepción de evaluación de los aprendizajes de las personas tutoras y el conocimiento que poseen del modelo pedagógico, identificar la concepción de evaluación de los aprendizajes y la percepción del modelo pedagógico de las personas encargadas de cátedra y de programa; por último determinar la congruencia de las propuestas evaluativas y los recursos didácticos con el modelo pedagógico. Se utilizaron:

Técnica: Análisis de contenido

Instrumento: Guías de revisión documental

- Recursos Didácticos.

- Orientaciones (instrumento 1). Consiste en cuatro secciones: estructura general del documento, materiales y apoyos didácticos, modelo de evaluación y descripción de los instrumentos de evaluación.

- Modelos de evaluación (instrumento 2). Cuadro de cotejo acerca de la índole de opciones evaluativas de las asignaturas seleccionadas, en el cual se constatan las modalidades en que se ofrece, los apoyos didácticos; la variedad y la cantidad de instrumentos empleados en la evaluación. 
Técnica: Encuesta

Instrumentos: Cuestionario y entrevista aplicados a:

- Personas tutoras del programa.

- Personas encargadas de cátedra.

- Encargado del programa I y II ciclos.

\section{Los participantes}

Para el desarrollo de la investigación se contó con la participación de 61 personas tutoras, nueve personas encargadas de las cátedra y el encargado del programa quienes pertenecen al diplomado, bachillerato y licenciatura en Educación General Básica I y II ciclos.

\section{- Las fuentes}

Se emplearon dos tipos de fuente: orales y documentales. Las orales conformadas por las personas participantes de la investigación, quienes aportaron para la obtención de información referente a otros objetivos de estudio que no han sido considerados aquí. El material impreso y electrónico de la UNED corresponde a las siguientes fuentes documentales (solamente las primeras se relacionan con esta publicación):

- Orientaciones académicas de las asignaturas: folleto por asignatura y por cuatrimestre que contiene objetivos, contenidos, instrumentos y técnicas de evaluación, instrucciones generales para el desarrollo de las tareas, proyectos, foros de discusión, estudios de casos, entre otros.

- Pruebas escritas aplicadas en las asignaturas del programa correspondientes a los periodos académicos del 2011 e inicios del 2012.

- $\quad$ Informe de Autoevaluación del Programa 2011.

- Investigación Evaluación del proceso de evaluación de los aprendizajes de la UNED.

- Modelo Pedagógico de la UNED.

- Investigación La evaluación de los aprendizajes una visión desde el contexto de los cursos de la Escuela de Ciencias de la Educación.
La metodología adoptada considera cinco momentos, la separación obedece específicamente a un ordenamiento en lo que a recolección de información respecta; solamente el primer momento se relaciona con el presente artículo.

- $\quad$ Primer momento: Análisis de la propuesta evaluativa de cada asignatura en contraste con las orientaciones y los recursos didácticos que se emplean.

- Segundo momento: Aplicación del cuestionario a personas tutoras.

- Tercer momento: Aplicación de entrevistas a personas encargadas de cátedra y del programa.

- Cuarto momento: Análisis de las pruebas escritas de cada asignatura.

- Quinto momento: Elaboración del informe escrito.

Para realizar la investigación se seleccionaron nueve asignaturas pertenecientes al Programa de Diplomado, Bachillerato y Licenciatura en I y II Ciclos de la Enseñanza General Básica, que son parte de la Escuela de Educación y no de las otras escuelas de la universidad (cuadro 1).

Los criterios utilizados para seleccionar la muestra fueron:

- Una asignatura por cada persona encargada de cátedra, excepto las pertenecientes a la cátedra que coordina una de las investigadoras.

- Opinión del estudiantado en cuanto a variedad de instrumentos de evaluación, pruebas escritas y disconformidad con los materiales didácticos. Opinión del estudiantado en relación con la inclusión en la propuesta evaluativa de actividades prácticas.

- Satisfacción del estudiantado con respecto a la asignatura.

Los criterios anteriores se obtuvieron al analizar el documento elaborado por el Programa de Autoevaluación Académica (PAA) en el año 2011. Cada una de las asignaturas fue examinada durante dos periodos académicos comprendidos entre los años 2011 y 2012, con el propósito de determinar la variedad de instrumentos empleados para evaluar el aprendizaje. 
CUADRO 1

Asignaturas seleccionadas y períodos académicos analizados

\begin{tabular}{|c|c|c|c|c|c|c|}
\hline Grado & Código & Asignatura & 2011-1 & 2011-2 & 2011-3 & 2012-1 \\
\hline Diplomado & 125 & Didáctica de las Ciencias Naturales & & $\mathrm{x}$ & & \\
\hline Diplomado & 108 & Recursos Audiovisuales & & $\mathrm{x}$ & $\mathrm{x}$ & \\
\hline Diplomado & 197 & Planeamiento Didáctico & & & $\mathrm{x}$ & $\mathrm{x}$ \\
\hline Diplomado & 767 & Introducción a la Pedagogía & & $\mathrm{x}$ & $\mathrm{x}$ & \\
\hline Bachillerato & 117 & Orientación Educativa & & & $\mathrm{x}$ & $\mathrm{x}$ \\
\hline Bachillerato & 114 & Sociología de la Educación & & $\mathrm{x}$ & $\mathrm{x}$ & \\
\hline Licenciatura & 442 & Métodos y Técnicas de Evaluación & & $\mathrm{x}$ & $\mathrm{x}$ & \\
\hline Licenciatura & 173 & Evaluación Institucional & $\mathrm{x}$ & & $\mathrm{x}$ & \\
\hline Licenciatura & 372 & Estadística Aplicada a la Educación & & & $\mathrm{x}$ & $\mathrm{x}$ \\
\hline
\end{tabular}

Fuente: Elaboración propia 2013.

Por tanto, se analizó la información contenida en las orientaciones académicas.

\section{RESULTADOS Y ANÁLISIS}

Se sistematizó y caracterizó la información contenida en el documento denominado Orientaciones Académicas, con lo cual se obtuvo la tabulación de las opciones evaluativas que se emplearon en cada asignatura, lo cual permitió realizar un análisis cuantitativo de los datos.

Con la aplicación del Instrumento 1 se indagó la existencia de los aspectos básicos que deben contener las orientaciones académicas, según el documento Lineamientos para la Evaluación de los Aprendizajes en la $U N E D$, al respecto se presenta la información relacionada con las asignaturas de la muestra (figura 1).

En la figura 1 se observa el cumplimiento total de la inclusión del propósito y del modelo de evaluación de los aprendizajes que se utiliza, por lo que queda claro que el estudiantado al matricular conoce el para qué y cómo será evaluado.

Por otra parte, una asignatura omite el cronograma y el nombre de los materiales, así el estudiantado se ve afectado en la organización del tiempo de estudio y el cumplimiento de las fechas de entrega de trabajos.

Dos aspectos no se incluyen en cuatro de nueve asignaturas: los objetivos de aprendizaje y las consideraciones generales o notas aclaratorias, en algunos casos los primeros se encuentran en las unidades didácticas y las segundas no son de carácter obligatorio.

Siete de las nueve asignaturas no incluyen los contenidos y las rúbricas. La primera omisión es justificada en forma escrita porque se mencionan en las unidades didácticas correspondientes, así de una u otra manera el estudiantado tiene acceso a tal información. El segundo tema es fundamental en la guía que debe poseer el estudiante en la realización de los trabajos, ya que se detallan los criterios con los cuales se calificaría su desempeño y evitaría la subjetividad.

En tres asignaturas no se menciona la metodología, lo cual puede afectar la comprensión que debe tener el estudiantado del rol del docente y la forma de desarrollarse.

Con el análisis de los temas básicos que incluyen la descripción de los proyectos, las tareas, los ensayos, entre otros, que para este caso se denominaron instrumentos de medición; se pudo apreciar que solamente tres, de las ocho asignaturas, emplean en la propuesta evaluativa un segundo instrumento de medición, diferente a la prueba escrita y únicamente dos asignaturas utilizan tres instrumentos. Además, se observa que una de las asignaturas incluye una tarea que no cumple con ninguno de los temas mencionados.

El total de las asignaturas de la muestra se brindan en modalidad tradicional, sin embargo, dos de ellas se ofrecen también en modalidad virtual. Congruente con 


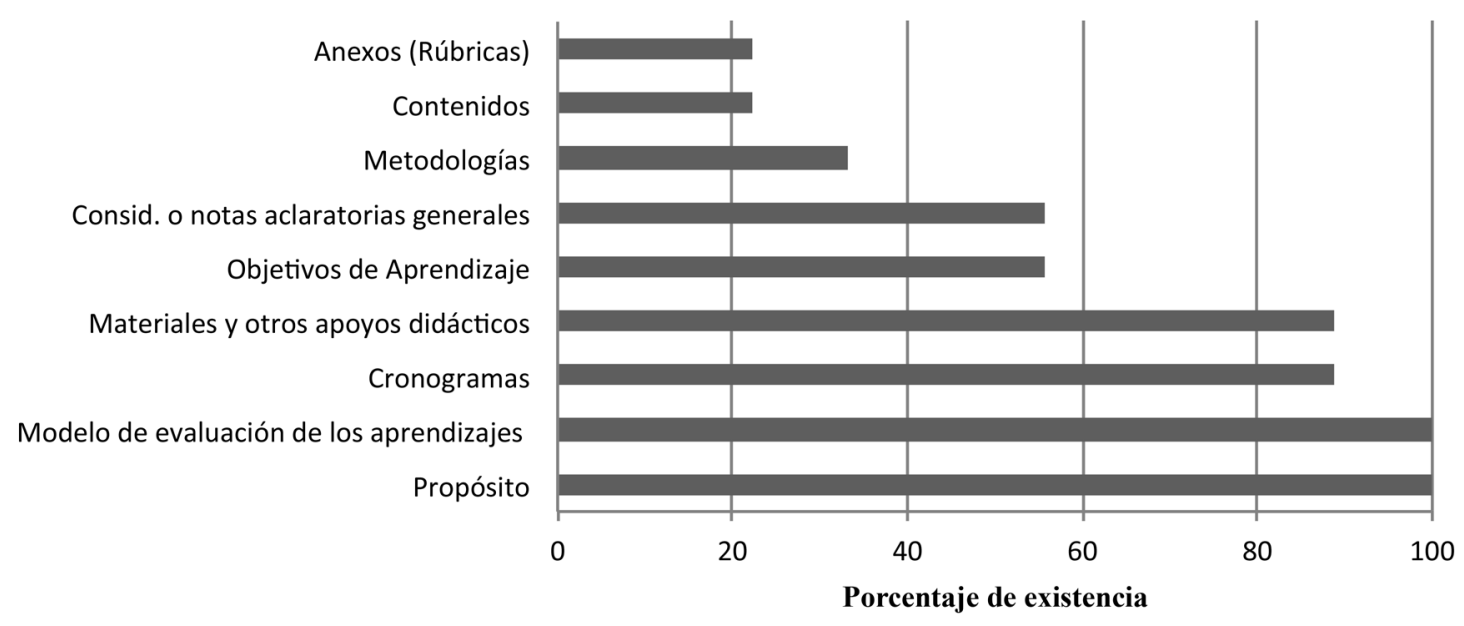

Fig. 1. Componentes básicos de las orientaciones académicas de acuerdo con los lineamientos de la Institución. Fuente: Elaboración propia con base en el Instrumento 1 (2012).

la modalidad tradicional, la mayoría de las asignaturas imparten tutorías presenciales, mínimo dos y máximo cuatro por periodo académico.

Con respecto a los apoyos resulta interesante que solo dos asignaturas utilizan la videoconferencia, ya que, el recurso se encuentra en la mayoría de los Centros Universitarios de la UNED, sin embargo se constata su poca utilización. Por otra parte, la universidad cuenta con otros apoyos didácticos como los multimedia educativos y los audiovisuales, pero en el análisis realizado tampoco se evidencia su empleo.

En cuanto al Programa de Apoyo Académico a Distancia (PADD), para la atención de consultas vía telefónica y por internet, se nota su utilización en todas las asignaturas analizadas.

En la figura 2, se distingue la variedad de opciones empleadas en la evaluación de las asignaturas.

De la información presentada en la figura 2 , se observa que en las propuestas evaluativas de siete asignaturas de la muestra se aplican variedad de instrumentos de medición diferentes a la prueba escrita; en una asignatura el modelo de evaluación está formado únicamente por dos pruebas y en otra no se aplican pruebas. Asimismo, en dos de las asignaturas se aplican foros de discusión, pero en una de ellas no se indica la cantidad de foros por realizar.
Con base en la información obtenida al analizar las orientaciones y aplicar el Instrumento 2 , se ha logrado identificar la índole de las opciones evaluativas propuestas por periodo académico en cada tarea o proyecto diferentes a la prueba escrita, las cuales se consignan por asignatura en el cuadro 2.

La información analizada muestra la tendencia por utilizar diferentes estrategias como comentarios de texto, mapas conceptuales, proyectos de investigación, análisis de datos, entre otros (no solo cuestionarios y resúmenes). Por otra parte, se observa que en ninguna asignatura se evalúa con prácticas docentes en el aula, registros anecdóticos, wikis, blogs, web-quest y búsqueda del tesoro.

Con la información recolectada se percibe que en algunas asignaturas se solicita la realización de una sola estrategia, por ejemplo: elaborar un ensayo o una investigación bibliográfica, contestar un cuestionario o crear una propuesta pedagógica; sin embargo, algunas veces también se requiere que el estudiantado desarrolle en una tarea o proyecto, una variedad de estrategias como la elaboración de un cuadro comparativo y un análisis de datos o un diseño de investigación y un ensayo, entre otros.

Además, se puede notar que una de las asignaturas no utiliza ninguna estrategia evaluativa diferente a la prueba escrita. 


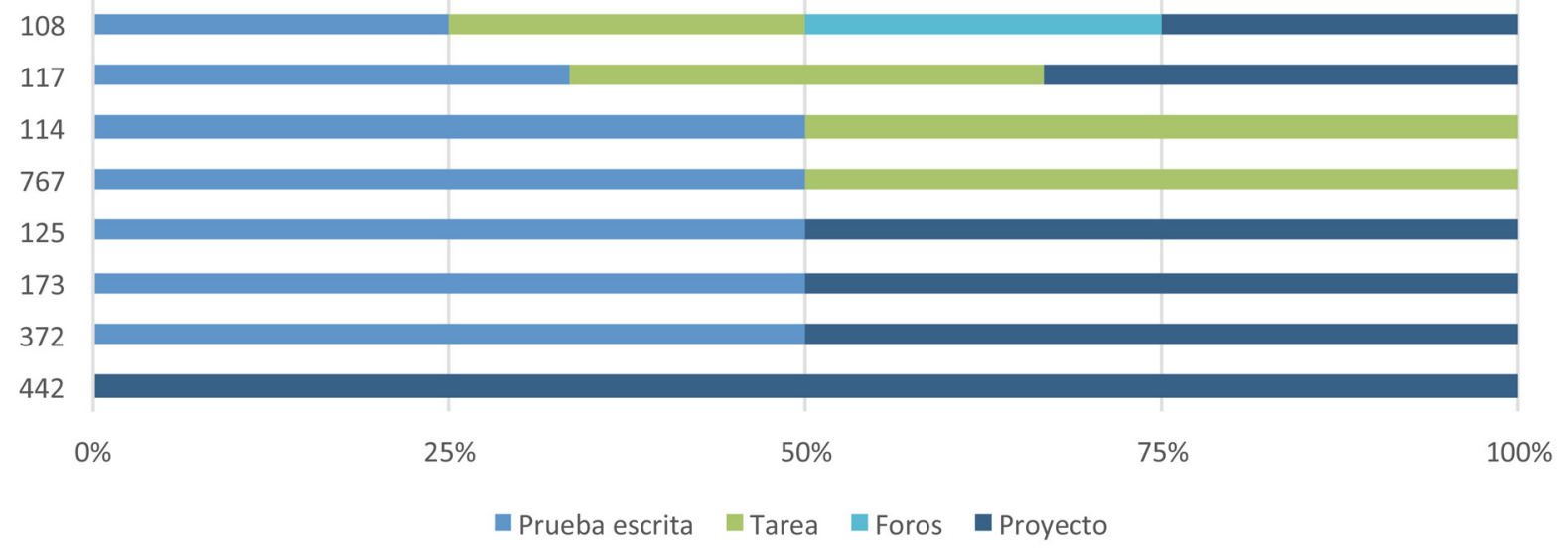

Fig. 2. Opciones evaluativas empleadas en las asignaturas seleccionadas durante los períodos académicos 2011-2012.

Fuente: Producción propia con base en el Instrumento 2 (2012).

CUADRO 2

Estrategias evaluativas empleadas en las asignaturas durante los períodos académicos 2011-2012

\begin{tabular}{l|llllll}
$\quad$ Estrategia de evaluación & \multicolumn{3}{c}{ Asignatura } \\
Comentario & 108 & 197 & 442 & 125 & 372 & 114 \\
Observación de aula & & & 117 \\
Diseño de investigación \\
Investigación bibliográfica
\end{tabular}

Fuente: Producción propia con base en el instrumento 2 (2012). 


\section{CONCLUSIONES}

Una vez analizados los resultados de la investigación se puede concluir que:

- Todas las asignaturas de la muestra incluyen el propósito y el modelo de evaluación en las orientaciones académicas.

- Se observó la ausencia de los objetivos específicos en algunas orientaciones académicas de las asignaturas, debido a que incluyeron los objetivos específicos en la unidad didáctica.

- En algunos casos los objetivos propuestos en las unidades didácticas no coinciden con los objetivos generales que fueron aprobados en los diseños curriculares.

- Todas las asignaturas de la muestra, ofertadas en modalidad tradicional, aplican una o dos pruebas escritas en su evaluación.

- La mayoría de las asignaturas incorpora al menos un instrumento de evaluación diferente de la prueba escrita, sin embargo, en algunos casos no está elaborado correctamente.

- Solamente una de las asignaturas evalúa por medio de dos pruebas escritas con un valor de $50 \%$ cada una.

- Seis de las nueve asignaturas solicitan la elaboración de un proyecto como instrumento de evaluación.

- Las asignaturas que se ofrecen en modalidad virtual incorporan foros y tareas como instrumentos de evaluación y no aplican pruebas escritas.

- No existen lineamientos claros en cuanto la extensión o cantidad de estrategias evaluativas que se deben incluir en una tarea o proyecto, esto queda a criterio de las personas encargadas de cátedra $y$, por tanto, el estudiantado debe esforzarse más en algunas asignaturas que en otras para lograr aprobarlas.

- Es recomendable utilizar el documento Lineamientos para la evaluación de los aprendizajes en la UNED con el propósito de homogenizar los aspectos que se incluyen en las orientaciones académicas; así como para contar con otras alternativas de evaluación.

- Tal como mencionan Padilla y Gil (2008):

De esta forma nos resultará posible revisar diferentes estrategias evaluativas a la luz de las condiciones, analizando qué hay que tener en cuenta en cada una de ellas para que su aplicación y su uso oriente al aprendizaje del alumno y no exclusivamente a la calificación. (p. 468)

Para mutar del concepto de evaluación tradicional del personal docente hacia el concepto de evaluar para aprender, no basta con cambiar las tradicionales pruebas escritas por otro instrumento de medición; más bien, es necesario orientar la evaluación para tratar de convertirla en un elemento optimizador del aprendizaje.

\section{REFERENCIAS}

Álvarez, L., González, P., González, J.A., Muñiz, J., Núñez, J.C., Rodríguez, T. y Soler, E. (2006). La evaluación de aprendizajes. Madrid: CCS.

Arce, R. y Chavarría, V. (2010). Informe final de investigación: Evaluación del Proceso de Evaluación de los Aprendizajes en la UNED. San José: EUNED.

Barrantes, R. (2002). Investigación un camino al conocimiento. Un enfoque cuantitativo y cualitativo. San José: EUNED.

Bordas, M. I., y Cabrera, F. (2001). Estrategias de evaluación de los aprendizajes centrados en el proceso. Revista española de pedagogía, 218(25-48)

Recuperado de http://cmapspublic3.ihmc.us/ rid=1GLSW84JS-WYZWX0-H40/Evaluaci\%C3\%83\%C6 \%92\%C3\%82\%C2\%B3n\%20del\%20Proceso\%20de\%20 Aprendizaje.pdf

Castillo, S. y Cabrerizo, J. (2003a). Evaluación educativa y promoción escolar. Madrid: Prentice Hall. Pearson.

Castillo, S. y Cabrerizo, J. (2003b). Prácticas de Evaluación Educativa. Madrid: Prentice Hall. Pearson.

Castillo, S. y Cabrerizo, J. (2010). Evaluación educativa de aprendizajes y competencias. Madrid: Prentice Hall. Pearson. 
Consejo Superior de Educación. (1994). Política Educativa Hacia el Siglo XXI. San José: MEP.

Hernández, S., Fernández, C. y Baptista, P. (2006). Metodología para la investigación. México: Mc Graw-Hill

Moreno Olivos, T. (2009). La evaluación del aprendizaje en la universidad. Tensiones, contradicciones y desafíos. Revista Mexicana de Investigación Educativa, 14(41) 563591. Recuperado de http://www.redalyc.org/articulo. oa?id=14004110

Carmona, M.T.P., y Flores, J.G. (2008). La evaluación orientada al aprendizaje en la Educación Superior: condiciones y estrategias para su aplicación en la docencia universitaria. Revista española de pedagogía, 467-485 Recuperado de http://dialnet.unirioja.es/descarga/articulo/2709011. pdf

Rivera, Y. (2003). Técnicas e instrumentos para la evaluación de los aprendizajes. Documento de trabajo para cursos. San José: EUNED.

Rivera, Y. y Sánchez, A. (2007). La evaluación de los aprendizajes: una visión desde el contexto de los cursos de la Escuela Ciencias de la Educación. San José: EUNED.

Universidad Estatal a Distancia (2001a). Jornada de reflexión y recomendaciones sobre la evaluación de los aprendizajes. Comisión de la Evaluación de los Aprendizajes. San José: UNED.

Universidad Estatal a Distancia (2001b). Factores de claves de éxito para el quinquenio 2001-2005. San José: EUNED.
Universidad Estatal a Distancia (2004). Modelo Pedagógico de la Universidad Estatal a Distancia. San José: EUNED.

Universidad Estatal a Distancia (2008). Plan Académico de la UNED 2008-2011. San José: EUNED.

Universidad Estatal a Distancia (2010a). Informe sobre evaluación de los aprendizajes en la Universidad Estatal a Distancia. Centro de Investigación y evaluación institucional. San José: EUNED.

Universidad Estatal a Distancia (2010b). Plan de Estudios Diplomado, Bachillerato y Licenciatura del Programa de ly Il Ciclos de la Enseñanza General Básica. San José: EUNED.

Universidad Estatal a Distancia (2011a). Evaluación de los cursos de la Escuela Ciencias de la Educación. San José: EUNED.

Universidad Estatal a Distancia (2011b). Informe de autoevaluación del Programa de l y Il ciclos de la Educación General Básica. San José: EUNED.

Universidad Estatal a Distancia, Comisión Institucional de Evaluación de los Aprendizajes. (2012). Lineamientos para la evaluación de los aprendizajes en la Universidad Estatal a Distancia.

Recuperado de: http://www.uned.ac.cr/Academica/ images/PACE/recursos/Evaluacion_de_los_ Aprendizajes__09-07-13_modificado.pdf

Universidad Estatal a Distancia. (2013). Plan Académico de la UNED 2012-2017. San José: EUNED. 
